\title{
Hertzian Magnetic Dipoles Model of a Quasi- isotropic radiation microstrip patch based Antenna-in- Package
}

\author{
Maria Bermudez Arboleda \\ CEMSE \\ KAUST \\ Thuwal, Saudi Arabia \\ maria.bermudezarboleda@kaust. \\ edu.sa
}

\author{
Kirill Klionovski \\ CEMSE \\ KAUST \\ Thuwal, Saudi Arabia \\ kirill.klionovski@kaust.edu.sa
}

Abstract - The Internet of Things applications require small wireless sensors with quasi isotropic radiation, so that their placement is orientation insensitive. This paper shows the design steps for a microstrip patch based quasi-isotropic Antenna-inPackage (AiP). The structure proposed is a hollow cube with six microstrip patch radiators, one on each of its faces. The cubes internal walls are metallized to not only act as ground planes for the microstrip patch antennas, but also provide the necessary shielding between the radiators and the future embedded electronics. Thus, the problem in hand is to find the optimal phases to be applied to each patch so that when all six are activated simultaneously, the obtained radiation pattern is quasi-isotropic. Given the complexity of the structure, a simplified model of Hertzian magnetic dipoles was used to determine the phase each patch was to be excited to achieve the closest to an isotropic radiator as possible. A measured gain variation of $6.5 \mathrm{~dB}$ has been achieved for the entire 3D sphere after fabrication of a prototype. Keywords-Antenna in package (AiP), quasi isotropic radiation, Hertzian magnetic dipoles, microstrip patch antenna.

\section{INTRODUCTION}

Wireless sensor networks (WSN) are based on the principle of many small agents collecting data in a large area. This agents should be embedded systems with the capability to communicate in any direction as they are randomly deployed in the area to be sensed. For this, orientation insensitive radiation patterns are the rule of thumb. The most common radiation pattern for these kind of devices is the omnidirectional one, like in dipole antennas [1]. But, there is a new interest in achieving quasi-isotropic radiation patterns that will increase the coverage area further. More so, this should be compatible with additively manufactured devices that implement the antenna structure in the package itself in an Antenna-in-Package (AiP) approach, saving at the same time space on the design and reducing the cost of fabrication.

In literature, the $\mathrm{AiP}$ designs with quasi-isotropic radiation patterns are mainly based on folded dipoles, and show a large flaw which is the lack of shielding of package core which is supposed to hold the electronics [2]-[3]. This causes interference between the embedded electronics and the antenna radiofrequency performance in such small dimensions by detuning the later.

This paper then focuses on achieving quasi-isotropic

\author{
Su Zhen \\ CEMSE \\ KAUST \\ Thuwal, Saudi Arabia \\ su.zhen@kaust.edu.sa
}

\author{
Atif Shamim \\ CEMSE \\ KAUST \\ Thuwal, Saudi Arabia \\ atif.shamim@kaust.edu.sa
}

radiation by simultaneously creating a shielded core to hold batteries, controlling circuits and sensors. To do so, six microstrip patch antennas assembled together in a 3D cube structure is designed and the aim is to find the right phases to be applied to each patch so that when simultaneously excited the AiP radiates quasi-isotropically. A simplified model of the structure where the microstrip patches are replaced by Hertzian magnetic dipoles is used in EM simulations to reduce the time of computation, then these results are compared with those of the microstrip patches. The measured results for a working prototype are in agreement with simulations, the gain variation of the radiation pattern in quasi-isotropic mode was $6.5 \mathrm{dBs}$.

\section{AIP DESIGN AND HERTZIAN MAGNETIC DIPOLE EQUIVALENCE}

In this work, isotropy is represented by the gain variation $\mathrm{GV}$ of the radiation pattern, this is defined as the difference between the highest and lowest gain, thus, a lower GV value is preferable. There is no standard definition for the maximum GV value which makes an antenna quasi-isotropic, but previously proposed radiators that are called quasi-isotropic have GV limit values of $7 \mathrm{~dB}$ [3], so this is the maximum value this paper aims for.

Fig.1 (a) shows the proposed design of the AiP. To simplify the visualization of the problem in hand a simplified model was established. An accurate representation of the microstrip patch antennas is the transmission line model which radiates like a Hertzian magnetic dipole [4]. In the simplification process, each microstrip patch will then radiate as if it was a loop of current orthogonally intersecting the corresponding face of the cube directed as seen in Fig.1 (b).

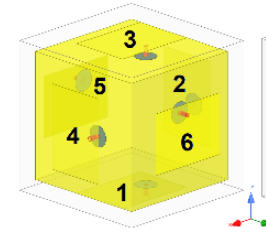

(a)

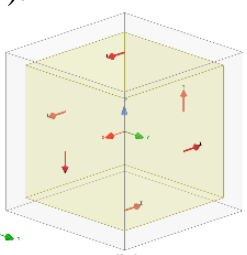

(b)

Fig. 1. (a) Proposed radiating structure. (b) Corresponding simplified Hertzian magnetic dipoles model.

For validation of each step, a simulation setup with Hertzian magnetic dipoles is carried in Ansys HFSS. As these are ideal 
sources, their radiation patterns are not presented in realized gain but in electric field intensity which is in direct correlation with gain [5]. The design evolution aims to find the minimum possible value for the variation in the electric field pattern for the whole sphere in the dipoles model. This minimum will then translate to the lowest GV in the microstrip patches design. The substrate used for simulation is 3D printable ABS, with measured permittivity of 4.8 , loss tangent of 0.008 and, a thickness of $4.5 \mathrm{~mm}$.

From the equivalence of the magnetic dipole, four co-planar dipoles in sequence, which are simultaneously excited with the same phase (red arrows in Fig.2 (a)), yield equal energy values in the corresponding plane. Given the lack of sources in the corresponding orthogonal plane, two nulls appear on it. Thus, an omnidirectional radiation pattern seen in Fig. 2(b). GV in the $\mathrm{XZ}$ plane was $1.78 \mathrm{~dB}$, while, the full pattern $\mathrm{GV}$ in this step was $54 \mathrm{~dB}$. To avoid the presence of these nulls, two extra dipoles are placed in the remaining faces, shown as blue arrows in Fig.2 (a), and they are excited in phase with the previous four. This displaced the obtained nulls from $\theta=0^{\circ}$ to $\theta=74^{\circ}$ and from $\Phi=90^{\circ}$ to $\Phi=98^{\circ}$ as shown in Fig. 2(c). The 3D pattern $\mathrm{GV}$ in this step was $17.6 \mathrm{~dB}$. Due to this reduction on $\mathrm{GV}$, further exploration with this configuration of dipoles is carried out, this time in the phases with which each dipole is excited.

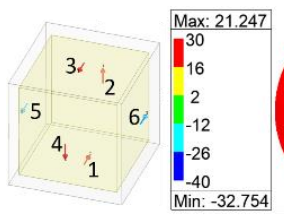

(a)

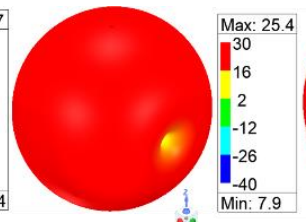

(b)

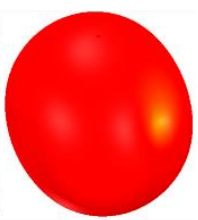

(c)
Fig. 2. Radiation pattern of modeled structure with Hertzian magnetic dipoles. (a) Basic structure with Hertzian dipoles vectors. (b) Electric field pattern in 3D for only dipoles 1,2,3 and 4 activated in phase. (c) Electric field pattern in 3D for all dipoles activated in phase.

Keeping the four primary dipoles in phase for the omnidirectional pattern, the value of phase $\psi$ for the two orthogonal dipoles placed in the $\mathrm{Y}$ axis was swept. The simulation results for the GV value in the whole 3D sphere are shown in Fig. 3(a). This phases were also applied to the microstrip patches design for corroboration. As can be observed, the behavior for microstrip patches is similar to that of the dipoles equivalence when different phases are applied to patches 5 and 6 (blue), but there is a small discrepancy in both the minimum achievable GV and the $\psi$ at which it happens. For the microstrip patches the minimum achievable $\mathrm{GV}$ is $4.4 \mathrm{~dB}$ compared with the $7.4 \mathrm{~dB}$ found for the dipoles, and it happens at $\psi=65^{\circ}$ compared to $\psi=60^{\circ}$.

This behavior can be attributed to the coupling effects $\left(S_{i j}\right)$ that rise with these kind of radiators when placed in arrays with low element spacing, as previously presented in [5]. Simulations were run to pinpoint the best side length of the cube as a compromise for size, coupling and lower GV, shown in Fig. 3(b). This resulted in an optimum side length of $\frac{\lambda_{0}}{2}$ which keeps the isolation value below $-15 \mathrm{~dB}$ and presents a $\mathrm{GV}$ of $4.4 \mathrm{~dB}$ in the simulations.

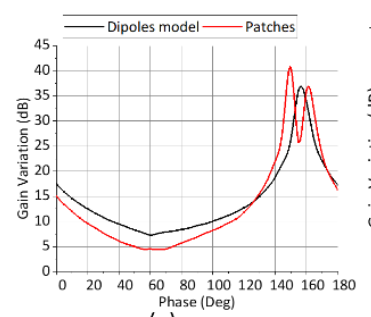

(a)

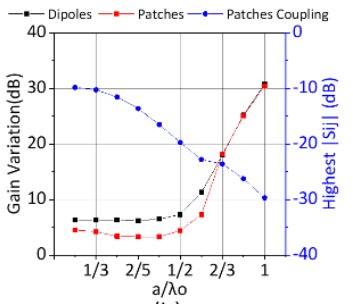

(b)
Fig. 3. (a) Change in GV of the electric field pattern due to phase shift applied to sources placed in $\mathrm{Y}$ axis for both dipoles and patches designs. (b) Effects of size reduction on $\mathrm{GV}$ of dipoles and patches structures. Coupling coefficient for the patches structure is also shown in blue.

Fig.4 (a) shows the built prototype of the structure. A feeding network needed to apply these found phases to each microstrip patch was placed inside the cube and the whole system was measured in Satimo anechoic chamber through a small cable opening in the bottom face which was previously simulated to confirm it did not affect the measured pattern. The measured radiation pattern shown in Fig.4 (b) presents a GV of $6.5 \mathrm{~dB}$ that although is higher than that expected in simulations, allows for this design to be considered a quasi-isotropic antenna. The discrepancy on GV can be attributed to surface roughness due to the $3 \mathrm{D}$ printing of the AiP substrate and losses in the connection points and feeding network used.

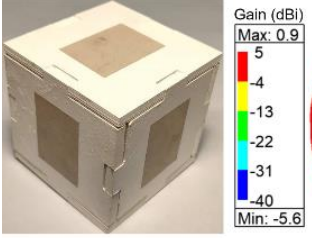

(a)

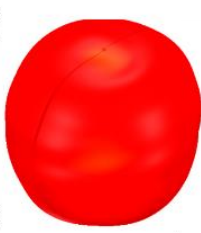

(b)
Fig. 4. (a) Fabricated prototype. (b) Measured radiation pattern of proposed structure.

\section{CONCLUSION}

In this work, we proposed a multi-radiator cubic AiP, which can provide quasi-isotropic radiation pattern. The tradeoffs between the size of the cubic AiP, inter element coupling and isotropy have been studied in a simplified model of the design by implementing Hertzian magnetic dipoles. A prototype has been fabricated through additive manufacturing and the measured results verify the design concepts.

\section{REFERENCES}

[1] D. I. Inc. (February 06, 2018, Nov. 4, 2019). How to Choose the Right Antenna for Your IoT Application. Available: https://www.digi.com/blog/post/how-to-choose-the-right-antenna-foryour-iot-appli

[2] Z. Su, K. Klionovski, R. M. Bilal, and A. Shamim, "A Dual Band Additively Manufactured 3-D Antenna on Package With Near-Isotropic Radiation Pattern," IEEE Transactions on Antennas and Propagation, vol. 66, no. 7, pp. 3295-3305, 2018.

[3] H. F. Abutarboush, M. F. Farooqui, and A. Shamim, "Inkjet-Printed Wideband Antenna on Resin-Coated Paper Substrate for Curved Wireless Devices," IEEE Antennas and Wireless Propagation Letters, vol. 15, pp. 20-23, 2016.

[4] C.A. Balanis,Antenna Theory Analysis And Deisng, Third Edition, 2005,pp. 811-843.

[5] E. Penard and J. Daniel, "Mutual coupling between microstrip antennas," Electronics Letters, vol. 18, no. 14, pp. 605-607, 1982. 